\title{
White matter tracts for the trafficking of neural progenitor cells characterized by cellular MRI and immunohistology: the role of CXCL12/CXCR4 signaling
}

\author{
Chiao-Chi V. Chen · Yi-Hua Hsu • D. M. Jayaseema • \\ Jeou-Yuan Joanne Chen • Dueng-Yuan Hueng • \\ Chen Chang
}

Received: 20 November 2013/Accepted: 1 April 2014/Published online: 26 April 2014

(C) The Author(s) 2014. This article is published with open access at Springerlink.com

\begin{abstract}
White matter tracts are important for the trafficking of neural progenitor cells (NPCs) in both normal and pathological conditions, but the underlying mechanism is not clear. The directionality of white matter is advantageous for molecules or cells to distribute over a long distance, but this feature is unlikely solely responsible for efficient migration. The present study hypothesizes that the efficient migration of NPCs into white matter is under the influences of neurochemical attraction-CXCL12/CXCR4 signaling, a major mechanism underlying the targeted migration of NPCs. To test this view, the present study investigated the effects of CXCL12 administration into the corpus callosum (CC) on the migratory behavior of transplanted NPCs. A living animal tracking platform based on MRI and a magnetic cell labeling technique was employed. The NPCs were magnetically labeled and then transplanted at the right end of the CC. CXCL12 was infused continuously at the left end. Migration of NPCs was monitored
\end{abstract}

Electronic supplementary material The online version of this article (doi:10.1007/s00429-014-0770-4) contains supplementary material, which is available to authorized users.

C.-C. V. Chen · Y.-H. Hsu · D. M. Jayaseema

J.-Y. J. Chen · C. Chang $(\square)$

N123, Institute of Biomedical Sciences, Academia Sinica, 128,

Section 2, Academia Road, Nankang, Taipei 11529, Taiwan

e-mail: bmcchen@ibms.sinica.edu.tw

D.-Y. Hueng $(\bowtie)$

Department of Neurological Surgery, Tri-Service General

Hospital, National Defense Medical Center, 325, Section 2,

Cheng-Kung Road, Neihu, 11490 Taipei, Taiwan

e-mail: hondy2195@yahoo.com.tw

\section{D.-Y. Hueng}

Department of Biochemistry, National Defense Medical Center,

Taipei, Taiwan repeatedly over a 7-day course using 3D gradient echo $\mathrm{T} 2 *$-weighted imaging. It was found that, CXCL12 induced NPCs to migrate up to $1,881 \mu \mathrm{m}$ from the graft whereas the spontaneous migration was mere $200 \mu \mathrm{m}$. CXCL12 induced migration that was nine times as efficient in the speed. The results indicate that the CXCL12/CXCR4 signaling may be a mechanism via which NPCs efficiently migrate along the white matter tracts. The study also presents a potential strategy for facilitating the targeted migration in NPC therapy for brain disorders.

Keywords SDF - CXCR4 - Stem cell - Migration · Tropism $\cdot$ White matter $\cdot$ Neurogenesis $\cdot$ Homing $\cdot$ Directed migration
Abbreviations
NPCs Neural progenitor cells
CC Corpus callosum
MPIO Micron-sized paramagnetic iron oxide
3D Three dimensional
T2*WI T2*weighted imaging
PB Prussian blue
APC Allophycocyanin
7-AAD 7-Aminoactinomycin-D
NDS Normal donkey serum
DAB Diaminobenzidine

\section{Introduction}

White matter tracts, which mainly involve transmitting neural signals from one brain region to another, are important for the trafficking of neural progenitor cells (NPCs) (Suzuki and Goldman 2003; Yang et al. 2009). 
Both endogenous and transplanted NPCs exhibit the tendency to take advantage of the white matter tracts for migration regardless of the condition being normal or pathological. Magnetic resonance imaging showed that, in neonatal rats, endogenous NPCs in the subventricular zone labeled by micron-sized paramagnetic iron oxide (MPIO) moved into the corpus callosum (CC) to reach the cortical and other regions within 7 days (Yang et al. 2009). Using immunohistological approaches, it was shown that transplanted NPCs migrated extensively in the corpus callosum, internal capsule, and hippocampal fiber tracts in the neonatal rat brain after being deposited into the striatum (Zhang et al. 2001). In the brain of ischemic stroke, transplanted NPCs migrated over the $\mathrm{CC}$ to reach the infarct site at an estimated speed of $360 \mu \mathrm{m} / \mathrm{day}$ (Kim et al. 2004).

Little is known regarding the mechanism via which NPCs tend to utilize the white matter tracts as a migration route. Structurally, white matter is highly directional for water diffusion, as indicated by diffusion tensor MRI (Assaf and Pasternak 2008). It is evident that white matter, as compared to the gray matter, is more advantageous to enable molecules or cells to distribute over a long distance. However, the migration speed of engrafted NPCs through the CC was estimated to be $50-70 \mu \mathrm{m} /$ day in the normal condition (Flexman et al. 2011). Such spontaneous migration is relatively slow, suggesting the directional diffusion is unlikely solely responsible for the faster migration reported in many of the related studies (Kim et al. 2004; Yang et al. 2009; Zhang et al. 2001).

The present study hypothesizes that efficient migration along the white matter structures is under the influences of neurochemical attraction. It is well established that chemotaxis via CXCL12/CXCR4 signaling mediates the targeted migration of NPCs toward brain lesion. Studies have indicated that the increased CXCL12 levels of the injured site form a concentration gradient that attracts cells bearing the receptor to migrate along (Tiveron et al. 2006; Liapi et al. 2008; Robin et al. 2006; Itoh et al. 2009; Imitola et al. 2004; Stumm and Hollt 2007; Belmadani et al. 2005, 2006; Bhattacharyya et al. 2008; Aboody et al. 2000; Banisadr et al. 2011; Takeuchi et al. 2007). NPCs, which extensively express the cognate receptor CXCR4 on the cell membrane, represent a major population being attracted by the CXCL12 elevation (Itoh et al. 2009; Peng et al. 2007). However, unlike the injured site, the white matter tracts are less concerned with regard to CXCL12 release in many of the studied conditions (white matter diseases being the exception). With little information available about CXCL12 expression in the fiber tracts, the role of the CXCL12/CXCR4 axis in the rapid migration through the white matter structure by NPCs remains to be investigated.
To the end, the present study employed a living animal tracking platform based on MRI and a magnetic cell labeling technique. The NPCs were magnetically labeled and then transplanted at the right end of the $\mathrm{CC}$ in adult rats. CXCL12 was infused continuously at the left end to form a concentration gradient. Migration was tracked by three-dimensional (3D) gradient echo $\mathrm{T} 2 *$ weighted imaging $(\mathrm{T} 2 * \mathrm{WI})$ repeatedly for up to 7 days. The temporal and spatial characteristics obtained from cellular MRI were used to characterize NPC migration along the CC. Immunohistology was also used to corroborate the MRI findings. The study used adult rats for investigations because minimal spontaneous NPC migration has been reported. The findings of the study present not only an understanding in the use of white matter as a migratory route by NPCs, but also a potential strategy for facilitating the targeted migration in NPC therapy for brain disorders.

\section{Materials and methods}

Animal subjects

Twenty-three adult male Sprague-Dawley rats purchased from National Laboratory Animal Center of Taiwan were used. Twelve rats were used in the first experiment, which was designed to validate the administration protocol (vehicle-treated, $n=6$; CXCL12-treated, $n=6$ ). Eleven rats were used in the cellular MRI experiment (vehicletreated, $n=5$; CXCL12-treated, $n=6$ ). The rats were 8-10 weeks old and weighed $250-300 \mathrm{~g}$ before experiments. Rats were housed in threes in plastic cages with free access to food and water. The housing environment was specific-pathogen-free with a 12:12-h light: dark cycle and controlled humidity and temperature. All experimental procedures were approved by the Institute of Animal Care and Utilization Committee at Academia Sinica, Taipei, Taiwan.

\section{Primary NPC culture}

Primary NPCs were harvested from D14 embryonic brain tissues of C57BL/6 pregnant mice purchased from National Laboratory Animal Center of Taiwan. The tissues were digested by collagenase type 2 (Sigma-Aldrich, St. Louis, MO, USA) at $2 \mathrm{mg} / \mathrm{mL}$ for $2 \mathrm{~h}$ at $37{ }^{\circ} \mathrm{C}$. The cells were plated at a density of $1.5 \times 10^{6}$ cells $/ \mathrm{mL}$ and propagated as free-floating neurospheres in the $\mathrm{N} 2$ medium consisting of Dulbecco's modified Eagle's medium and Ham's F-12 medium (DMEM/F-12) and 1 M HEPES (both from Gibco, Invitrogen, Grand Island, NY, USA), $0.285 \%$ D-glucose, $23 \mu \mathrm{g} / \mathrm{mL}$ insulin, $92 \mu \mathrm{g} / \mathrm{mL}$ apo-transferrin, $8.9 \mu \mathrm{g} / \mathrm{mL}$ putrescine, and $75 \mu \mathrm{M}$ sodium selenite (Sigma-Aldrich), 
$20 \mathrm{ng} / \mathrm{mL}$ human recombinant epidermal growth factor (BD Biosciences, San Jose, CA, USA), and $20 \mathrm{ng} / \mathrm{mL}$ FGF-2 (Sigma-Aldrich). Upon reaching of the sphere size to $140-150 \mu \mathrm{m}$, the cells were passaged by mechanical trituration with a fire-polished Pasteur pipette (Corning, Tewksbury, MA 01876, USA) through a $40-\mu \mathrm{m}$ cell strainer (BD Biosciences). The standard cell culture atmosphere was maintained with $5 \% \mathrm{CO}_{2}$ at $37{ }^{\circ} \mathrm{C}$ throughout the experiments.

\section{Flow analysis}

Aliquots of $1 \times 10^{6}$ viable cells were incubated in PBS with anti-mouse CXCR4 (1:100; eBioscience, San Diego, CA, USA) antibody conjugated with allophycocyanin (APC) and $1 \%$ fetal bovine serum (FBS) (Gibco) for 15 min in dark at $4{ }^{\circ} \mathrm{C}$. After three washes, the cells were resuspended in PBS containing $2.5 \mu \mathrm{g} / \mathrm{mL}$ of 7 -aminoactinomycin-D (7-AAD; BD Biosciences) and $1 \%$ FBS for $10 \mathrm{~min}$ at room temperature. Flow cytometry was performed on FACSCanto (BD Biosciences). A total of 10,000 counts were acquired for each analysis using FACS diva $\mathrm{v}$ 6.1.2 (BD Biosciences). The experiment was repeated three times.

\section{Magnetic NPC labeling with MPIO}

For magnetic labeling, NPCs were suspended in PBS with $150 \mu \mathrm{g} / \mathrm{mL}$ MPIO $(0.96 \mu \mathrm{m}$; catalog \# MC03N; Bangs Laboratories, Inc., Fishers, IN, USA) and then received electroporation of a single pulse at $0.17 \mathrm{~V}$ for $200 \mathrm{~ms}$ followed by overnight incubation using Gene Pulser (Bio Rad, Richmond, CA, USA). After three washes to remove free MPIO, NPCs were counted and used for experiments. For each labeling, $1 \times 10^{5}$ cells were spared to verify the labeling procedure using Prussian blue (PB) staining as described below. On average, $81.7 \pm 2.7 \%$ (mean \pm standard deviation) of the cells were positively labeled with MPIO.

\section{NPC engraftment and CXCL12 infusion}

The rats were anesthetized intraperitoneally by chloral hydrate (Sigma-Aldrich) at $450 \mathrm{mg} / \mathrm{kg} .1 \times 10^{6}$ MPIOlabeled NPCs in a volume of $5 \mu \mathrm{L}$ were injected into the right end of the CC (AP $1.0 \mathrm{~mm}$; $\mathrm{ML}+1.2 \mathrm{~mm}$; DV $-3 \mathrm{~mm}$ ) of the brain on a stereotaxic apparatus (Stoelting, Wood Dale, IL, USA). The injection rate was $1 \mu \mathrm{L} / \mathrm{min}$ by a micro-infusion pump (Model: Pump 11 Elite from Harvard Apparatus, Holliston, MA, USA). In addition, a PE-10 tube (BD Biosciences) ensheathed by PE50 connected to an osmotic minipump (Model \# 1002; ALZET Osmotic Pumps, Cupertino, CA, USA) was implanted at the left end
(AP $1.0 \mathrm{~mm}$; ML $-1.5 \mathrm{~mm}$; DV $-3 \mathrm{~mm}$ ). The minipump was filled with $100 \mu \mathrm{L}$ of solution with or without CXCL12 $(1 \mu \mathrm{g})$. The vehicle solution was PBS with $0.1 \%$ bovine serum albumin. The residual amount in the minipump was checked on D7 to ensure the delivery. The Alzet \#1002 minipump delivers $0.25 \mu \mathrm{L}$ every hour, and the residue was approximately $50 \mu \mathrm{L}$ on D7.

The administration approach of CXCL12 (Millipore) to the targeted region was verified using immunohistology in twelve rats. Six received CXCL12 minipump infusion, while the other received vehicle. The infusion dosage of CXCL12 was $60 \mathrm{ng} /$ day. This concentration was determined empirically accordingly to our pilot study. The dosage was much lower as compared to those of the previous studies (Shyu et al. 2008; Shin et al. 2011), which used a daily dosage of up to $4 \mu \mathrm{g}$. A possible explanation for a lower concentration was our use of minipump for continuous infusion. Given the fact that CXCL12 has a very short half-life $(25.8 \pm 4.6 \mathrm{~min})$ in the circulation (Rempel et al. 2000), bolus injection might be less effective than slow delivery and thus required much higher dosage.

PB staining in NPCs

$1 \times 10^{5}$ cells were spread on the glass slide by cytocentrifugation (Model: Cytospin-4, Shandon, Thermo Scientific, Asheville, NC, USA). After air drying, cells were fixed with $4 \%$ paraformaldehyde (Sigma-Aldrich), washed, incubated for 30 min with $10 \%$ potassium ferrocyanide (Sigma-Aldrich) in $20 \% \mathrm{HCl}$, washed again, and counterstained with nuclear fast red (Sigma-Aldrich). The slides were then dehydrated with 70,95 , and $100 \%$ ethanol for $1 \mathrm{~min}$ in each, cleared in two changes of xylene for 3 min, and mounted using DPX (Sigma-Aldrich). The slides were imaged under bright field at $100 \times$ magnification, and cells exhibiting blue intracellular particles were considered positive.

\section{The proliferation assay in NPCs}

MPIO-labeled and unlabeled NPCs were cultured in 96-well plates (BD Biosciences) at a density of 1,000 cells per well in $100 \mu \mathrm{l}$ of the $\mathrm{N} 2$ medium for 7 days. Ten microliter of MTT (Roche Applied Science, Indianapolis, IN, USA) in PBS at $5 \mathrm{mg} / \mathrm{mL}$ was added to each well. After $4 \mathrm{~h}$ of incubation, $100 \mu \mathrm{l}$ of a $10 \%$ (w/v) sodium dodecyl sulfate solution (Roche Applied Science) in $0.01 \mathrm{~N} \mathrm{HCl}$ was added to lyse the cells and to solubilize the formazan crystals. After $12 \mathrm{~h}$ of dissolution of the formazan crystals, the absorbance of the formazan product was measured using a Spectra Max 340PC384 microplate spectrophotometer (Molecular Devices, Downingtown, PA, USA) at a wavelength of $570 \mathrm{~nm}$ with $750 \mathrm{~nm}$ as the 
reference. The assay was repeated three times in quadruplicates.

The differentiation assay in NPCs

$1 \times 10^{4}$ labeled and unlabeled NPCs were seeded onto 12-mm coverslips (Paul Marienfeld GmbH \& Co. KG, Lauda-Königshofen, Germany) coated with $20 \mu \mathrm{g} / \mathrm{mL}$ of laminin (Millipore, Billerica, MA, USA). Differentiation was induced by growing the cells in $1 \%$ FBS supplemented N2 medium devoid of EGF and FGF. After 7 days, cells were fixed with $4 \%$ paraformaldehyde for 20 min and stained by neuronal, astrocyte, and oligodendrocyte markers. After blocking of nonspecific staining, the NPCs were incubated overnight at $4{ }^{\circ} \mathrm{C}$ with one of the marker antibodies, including rabbit anti-monoclonal GFAP (1:200 dilution, Abcam, Cambridge, MA, USA), mouse anti-Oligodendrocyte (1:200 dilution, Millipore, USA), and mouse anti-beta III tubulin (1:200 dilution, AbD Serotec, Raleigh, NC, USA). This was followed by incubation with secondary antibodies (donkey anti-rabbit or donkey antimouse from Jackson Immunoresearch Laboratories, West Grove, PA, USA) conjugated with rhodamine for $1 \mathrm{~h}$ in dark. The antibodies were diluted in PBS containing $10 \%$ normal donkey serum (NDS) (Sigma-Aldrich) and $0.1 \%$ Triton-X-100 (Fisher Scientific, Pittsburgh, PA, USA). The labeled cells were further stained with $\mathrm{PB}$ enhanced by DAB (Sigma-Aldrich). The PB-stained cells were reacted with $0.014 \%$ diaminobenzidine (DAB) activated by $0.03 \%$ hydrogen peroxide (Merck, Whitehouse Station, NJ, USA) for $5 \mathrm{~min}$.

In vivo MRI tracking of the implanted cells in the brain

Eleven rats engrafted with MPIO-labeled NPCs were used for MRI. Six were treated with CXCL12 while five with vehicle. The migration of the engrafted cells along the callosal pathway was tracked by $3 \mathrm{D} \mathrm{T} 2 *$-WI repeatedly at D0 (the engraftment day), D1, D3, and D7. Six CXCL12treated and five vehicle-treated animals were scanned. For MRI, each rat was anesthetized by $2 \%$ isoflurane in oxygen flowed at 1-2 L/min. MR images were acquired using a 4.7-T spectrometer (Biospec 47/40, Bruker, Karlsruhe, Germany) with a 72-mm volume coil as the RF transmitter and a quadrature surface coil placed on the head as the receiver. Rats are fixed in a prone position during scanning. A 3D gradient echo sequence was used with time of repetition $=30.0 \mathrm{~ms}$, time to echo $=15.0 \mathrm{~ms}$, flip angle $=$ $30^{\circ}$, number of average $=4$, field of view $=25.6 \times 25.6 \times$ $25.6 \mathrm{~mm}$, bandwidth $=45,045 \mathrm{~Hz}$, matrix $=256 \times 128 \times$ 128 zero-filled to $256 \times 256 \times 256$, and total scan time $=32 \mathrm{~m} 46 \mathrm{~s}$.
MRI data analysis

The migration distance and volume were calculated on T2*-WI using Avizo (Visualization Sciences Group 3D, Burlington, MA, USA). The pixels showing hypointensity in contrast to the background were selected by thresholding at the lowest $25 \%$ of the signal intensities and used for measurement. The distance or volume along the callosal pathway was defined as the distance or area from the edge of the graft proximal to the CXCL12 infusion pump to the end of the migration path toward the infusion site. The distance of the migratory path was calculated from an average of 6 slices covering the graft. The volume of the migratory path was the total summed voxel size from the defined migratory path.

Histology and immunohistology

The animals were perfused with $4 \%$ paraformaldehyde. The brain was sectioned at a thickness of $50 \mu \mathrm{m}$. For PB staining, the sections were treated with $2 \%$ (w/v) potassium ferrocyanide solution mixed with $2 \mathrm{~N} \mathrm{HCl}$ at a 1:1 ratio for $15 \mathrm{~min}$ and then counterstained with nuclear fast red (Sigma-Aldrich) for $3 \mathrm{~min}$. For immunohistology, after being pretreated with PBS containing $0.3 \% \mathrm{H}_{2} \mathrm{O}_{2}$ and $0.1 \%$ NaN3 (Sigma-Aldrich), the sections were incubated with mouse anti-CXCL12 (1:100; MAB\#350, R\&D systems, Minneapolis, MN, USA) or mouse anti-nestin (1:100) diluted in PBS containing $0.3 \%$ Triton-X and $5 \%$ normal donkey serum overnight at $4{ }^{\circ} \mathrm{C}$, followed by incubation in biotin-conjugated donkey anti-mouse $\operatorname{IgG}$ (1:200; Dako, Glostrup, Denmark) for $1 \mathrm{~h}$. The sections were immersed in PBS with $0.3 \%$ Triton-X and avidin-biotin complex (1:500) (Vector Labs, Burlingame, CA, USA) at room temperature for $3 \mathrm{~h}$. The sections were then stained in $0.05 \mathrm{M}$ Tris buffer with $0.025 \%(\mathrm{w} / \mathrm{v}) \mathrm{DAB}, 1.5 \%(\mathrm{w} / \mathrm{v})$ nickel ammonium sulfate (Honeywell Riedel-de Haen, Seelze, Germany) (omitted when staining nestin) and $0.024 \% \mathrm{H}_{2} \mathrm{O}_{2}$ for 2-5 min until the desired dark-purple color had developed. The sections were then washed, mounted on coated slides, dehydrated, and coverslipped with DPX. The CXCL12-stained sections were photographed under a light microscope (BX51, Olympus, Japan). CXCL12 immunoreactivity was observed as particles surrounding cells or in cells per se. The quantification was done by counting the number of particles that are stained positive for CXCL12.

Statistical analysis

The differences between CXCL12- and vehicle-treated groups were compared using $t$ tests or repeated-measure ANOVAs followed by Fisher's post hoc tests for multiple 
Fig. 1 CXCL12 levels assessed in the spontaneous and treated conditions. a A brain slice showing the minipump tubing tract and cell graft. b The CXCL12-positive particles surrounding cells were counted. For the CXCL12-treated group, CXCL12 immunoreactivity was significantly elevated at the infusion site after 1 day of infusion and increased further after 3 days. c CXCL12 infusion caused significant immunoreactivity nearby the infusion site. d Little immunoreactivity was observed at the graft site. The spontaneous and treated groups contained six rats each
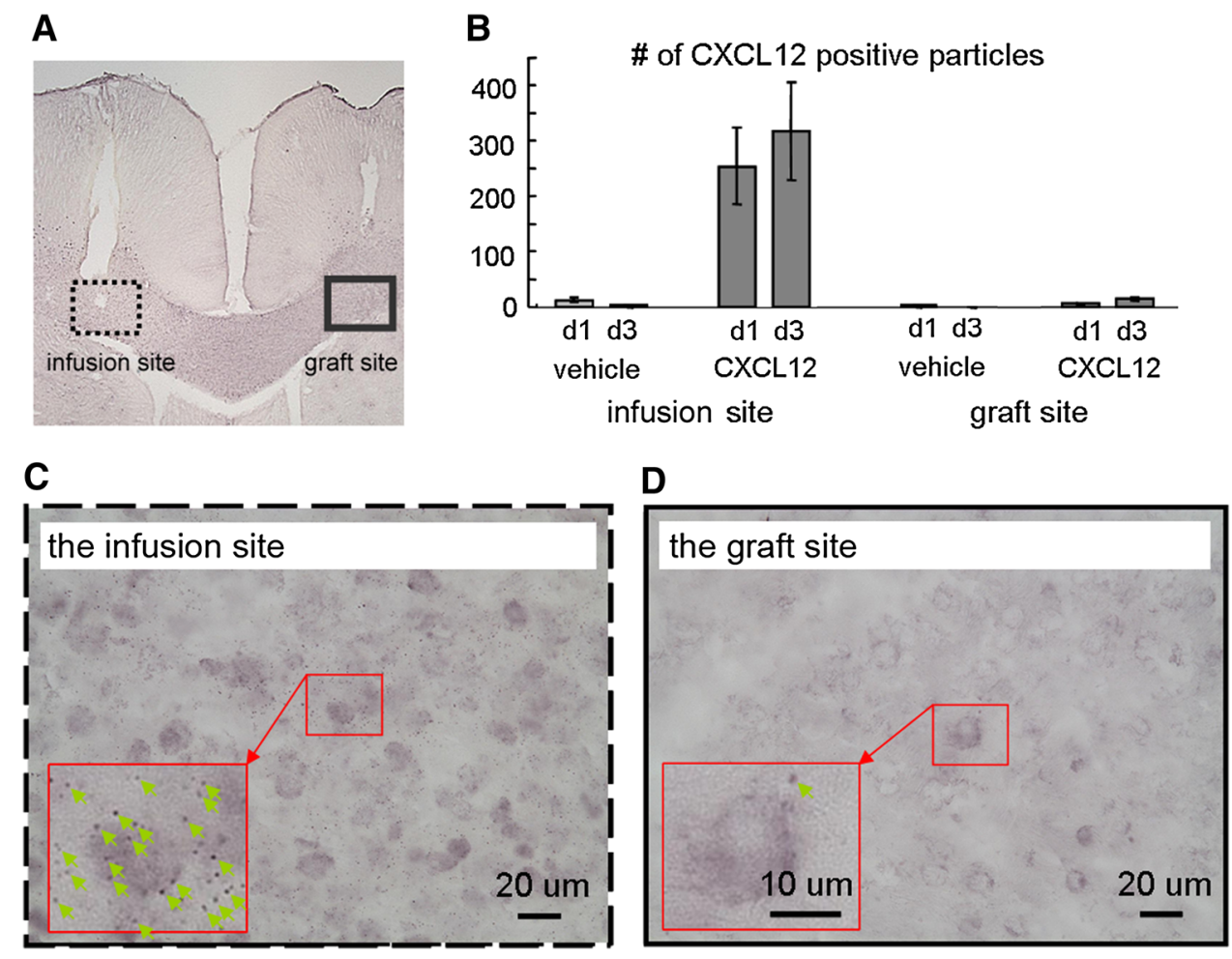

D comparison. The level of statistical significance was set at $p<0.05$.

\section{Data presentation}

The image data (photomicrographs in Figs. 1a, c, 5, 6; MR images in Fig. 3) are from single representative animals from each of the groups, while the group-averaged data are shown in Figs. $1 b$ and 4.

\section{Results}

CXCL12 levels assessed in the spontaneous and treated conditions

Figure 1a presents a brain slice showing the NPC graft and the CXCL12 infusion tract. Figure $1 \mathrm{~b}$ presents the quantitative data of CXCL12 immunoreactivity expressed by the count of the CXCL12-positive particles at the infusion and graft sites between the groups across time. In the spontaneous (vehicle-treated) condition, the CXCL12 level was minimal in the $\mathrm{CC}$ of the adult rat. By contrast, in the CXCL12 infusion condition, CXCL12 levels were significantly higher at the infusion site after 1 day of infusion and showed an increasing trend after 3 days. Figure $1 \mathrm{c}$ and $\mathrm{d}$ shows the magnified views of the infusion site and the graft site of the CXCL12 infusion condition, respectively. More immunoreactivity was observed at the infusion site. Many cells were surrounded by particles immunoreactive for CXCL12.

CXCR4 expression in NPCs verified by flow cytometry

The NPCs were stained by the antibody against CXCR4 and 7-AAD as the viability marker. Gating was done on unstained NPCs as shown in Fig. 2a. This generated four divisions as denoted. Figure $2 \mathrm{~b}$ shows that the majority of the stained NPCs fell within Q4 (CXCR4+/7AAD-). The viable, CXCR4-positive population was more than $95 \%$ as shown in Fig. 2c.

Cellular MRI shows CXCL12 induced stronger migration of NPCs along the CC in vivo

Axial 3D T2*-WI images were acquired on D0, D1, D3, and D7 after the transplantation and infusion surgery. Figure 3a shows the representative images at the four different time points merged from four slices that covered the CXCL12 infusion site and the migration path. The infusion minipump tubing appeared on the left while the NPC graft on the right as labeled. On D1, a stream-like hypointensity directed toward the side of the infusion tube was observed. On D3, the migratory path extended further from the NPC graft site. On D7, the path became more obvious due to increased hypointensities. In contrast, no obvious signal 
Fig. 2 CXCR4 expression in NPCs verified by flow cytometry. a Gating for positive or non-fluorescence was done on unstained NPCs. The levels of the fluorescence were minimal. $\mathbf{b}$ The majority of the NPCs stained with the CXCR4 antibody and 7AAD fell within Q4 (CXCR4+/7AAD-).

c More than $95 \%$ of the NPCs were viable and CXCR4 positive. The values are expressed as mean \pm standard deviation
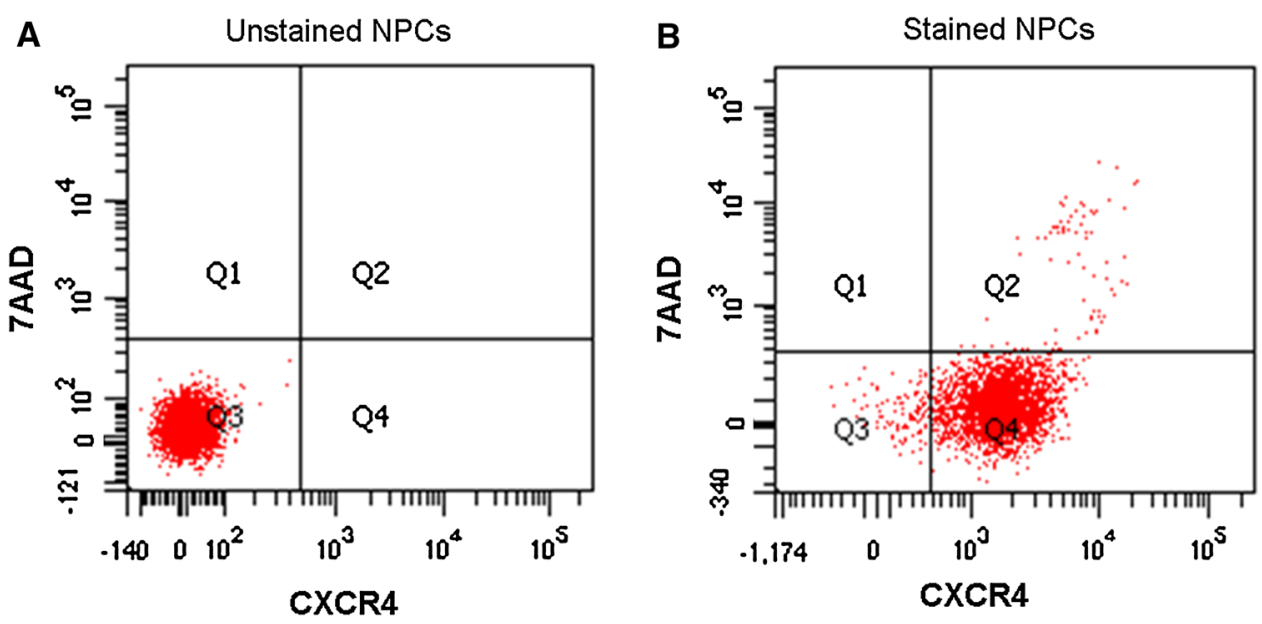

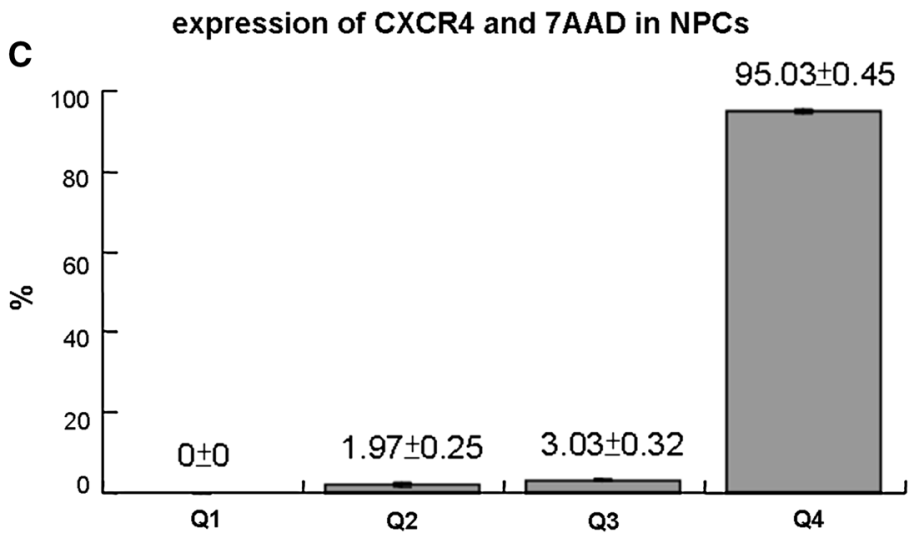

\section{A CXCL12}
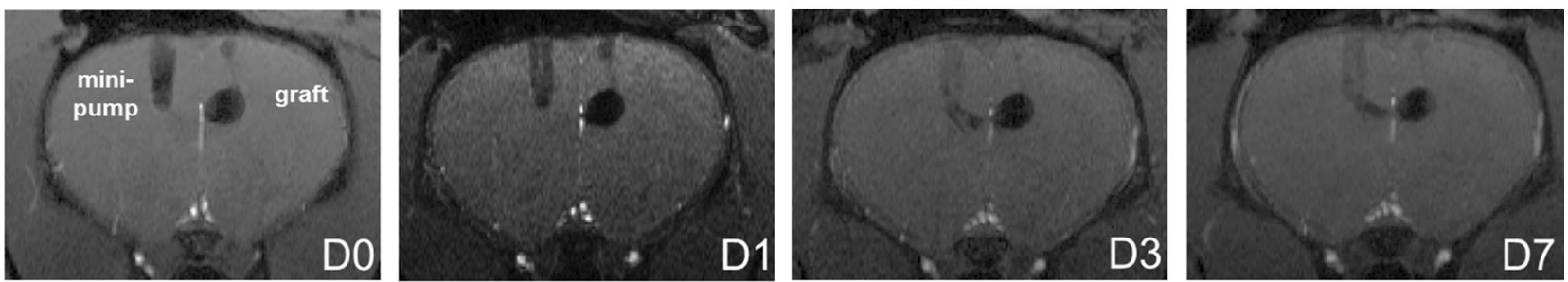

\section{B vehicle}
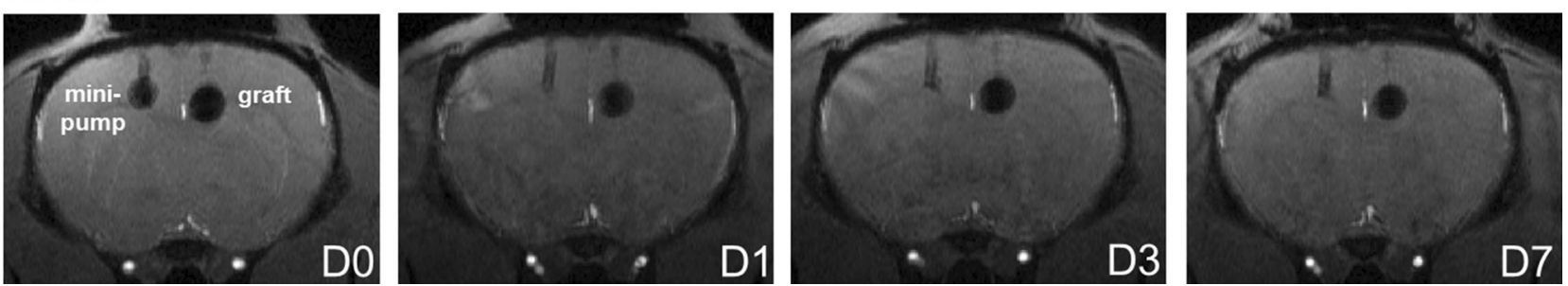

Fig. 3 Cellular MRI shows CXCL12 induced stronger migration of NPCs along the CC in vivo. a The infusion tube appeared on the left while the NPC graft on the right as labeled. On D1, CXCL12 infusion led to a slight hypointense stream directed toward the side of the infusion tube. On D3, the migratory path extended from the NPC graft toward the infusion site. On D7, the path became more visible due to increased hypointensities. b Infusion of vehicle induced no obvious signal change along the callosal pathway with time. The vehicle- and CXCL12-treated groups contained five and six rats, respectively 

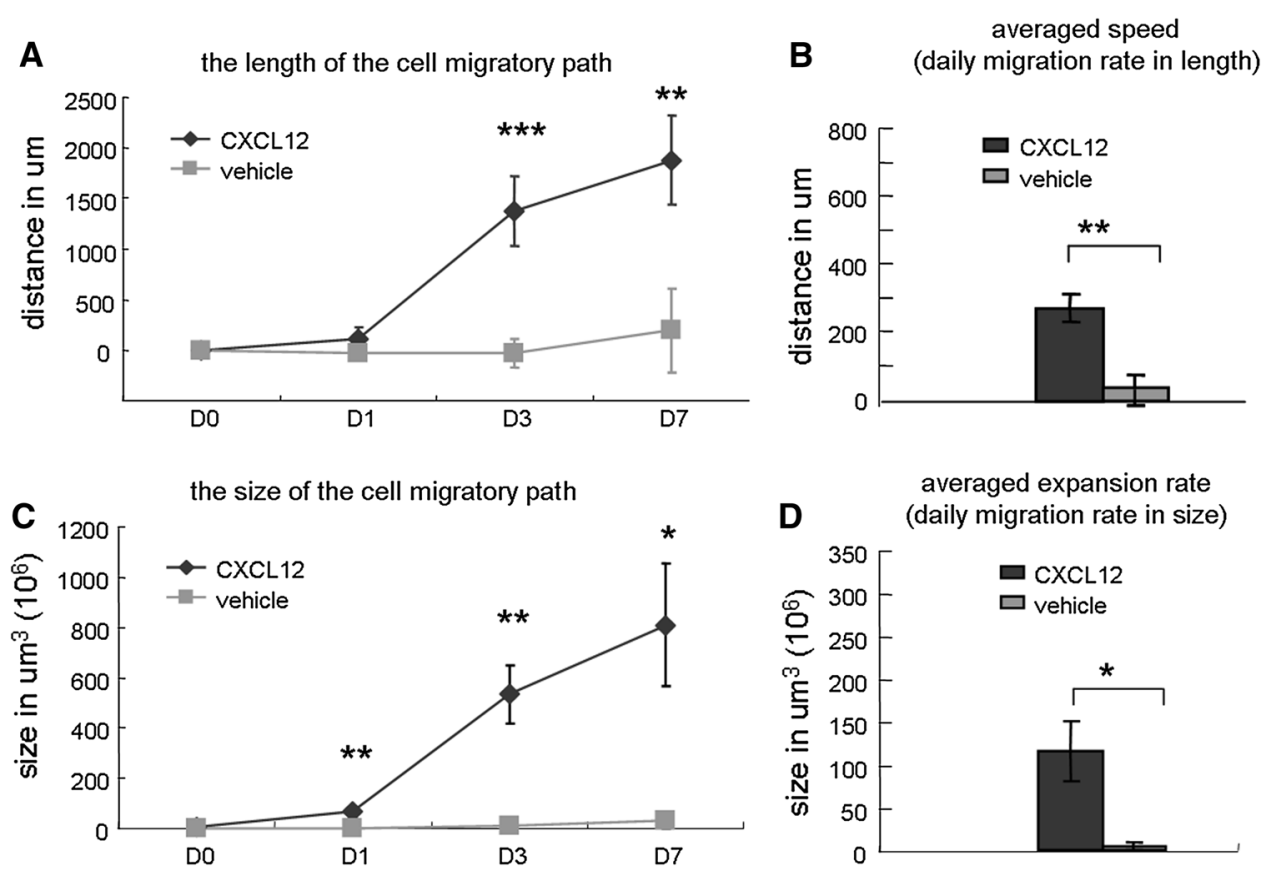

Fig. 4 Measuring spontaneous and induced migration along the $\mathrm{CC}$ on cellular MRI. a In length, CXCL12 induced the NPCs to migrate with time over an extensive range from the grafted area. CXCL12induced migration was nearly nine times as long as the vehicleinduced migration. b The averaged migration speeds were $269 \pm 41$ and $29 \pm 45 \mu \mathrm{m} /$ day for the CXCL12 and vehicle groups, respectively. c The migratory path increased in size with time. The size of CXCL12-induced migration was 28.7 times as large as vehicle-induced migration (i.e., spontaneous). d The average expansion rates from D0 to D7 were $115 \pm 35 \times 106$ and $4 \pm 4 \times$ $106 \mu^{3} /$ day for the CXCL12 and vehicle groups, respectively

$p<0.01]$. The averaged migration speeds, calculated from the migration distance over time, were $269 \pm 41$ and $29 \pm 45 \mu \mathrm{m} /$ day for the CXCL12 and vehicle groups, respectively ( $p<0.01)$ (Fig. $4 b)$.

The migratory path was found to increase in size with time as shown in Fig. 4c. The size of CXCL12-induced migration was 28.7 times as large as that of the vehicleinduced (i.e., spontaneous). The statistical differences were detected by repeated-measures ANOVA followed by Fisher's post hoc tests $[F(1,9)=12.57, p<0.01$; D1, $p<0.01 ; \mathrm{D} 3, p<0.01 ; \mathrm{D} 7, p<0.05]$. The expansion rates, calculated from the size increments over time, are shown in Fig. 4d. The averaged expansion rates from D0 to D7 were $115 \pm 35 \times 10^{6}$ and $4 \pm 4 \times 10^{6} \mu^{3} /$ day for the CXCL12 and vehicle groups, respectively $(p<0.05)$.

\section{Corroboration of the chemotaxis by PB staining}

PB staining was used to confirm the presence of MPIOlabeled NPCs in the brain sections. NPC migration induced by CXCL12 infusion is visible as hypointensities on T2*WI (Fig. 5a). Figure 5b depicts the distribution of PBstained cells corresponding to the graft, migratory path, and the minipump. The PB staining matched with the hypointensities on T2*-WI. Magnified views of the migratory path 
Fig. 5 Corroboration of the chemotaxis by PB staining. a The NPC migration induced by CXCL12 infusion seen as the hypointensities on $\mathrm{T} 2 * \mathrm{WI}$. b The PB staining concurred with the hypointensities on T2*WI. c A magnified view of the migratory path toward the target. d A view of the graft. e $\mathrm{T} 2 * \mathrm{WI}$ of the group with vehicle infusion showed little migration. f PB-stained cells were mainly distributed in the graft site. $\mathrm{g}$ A magnified view of the corpus callosum. $\mathbf{h}$ A view of the graft

\section{CXCL12}
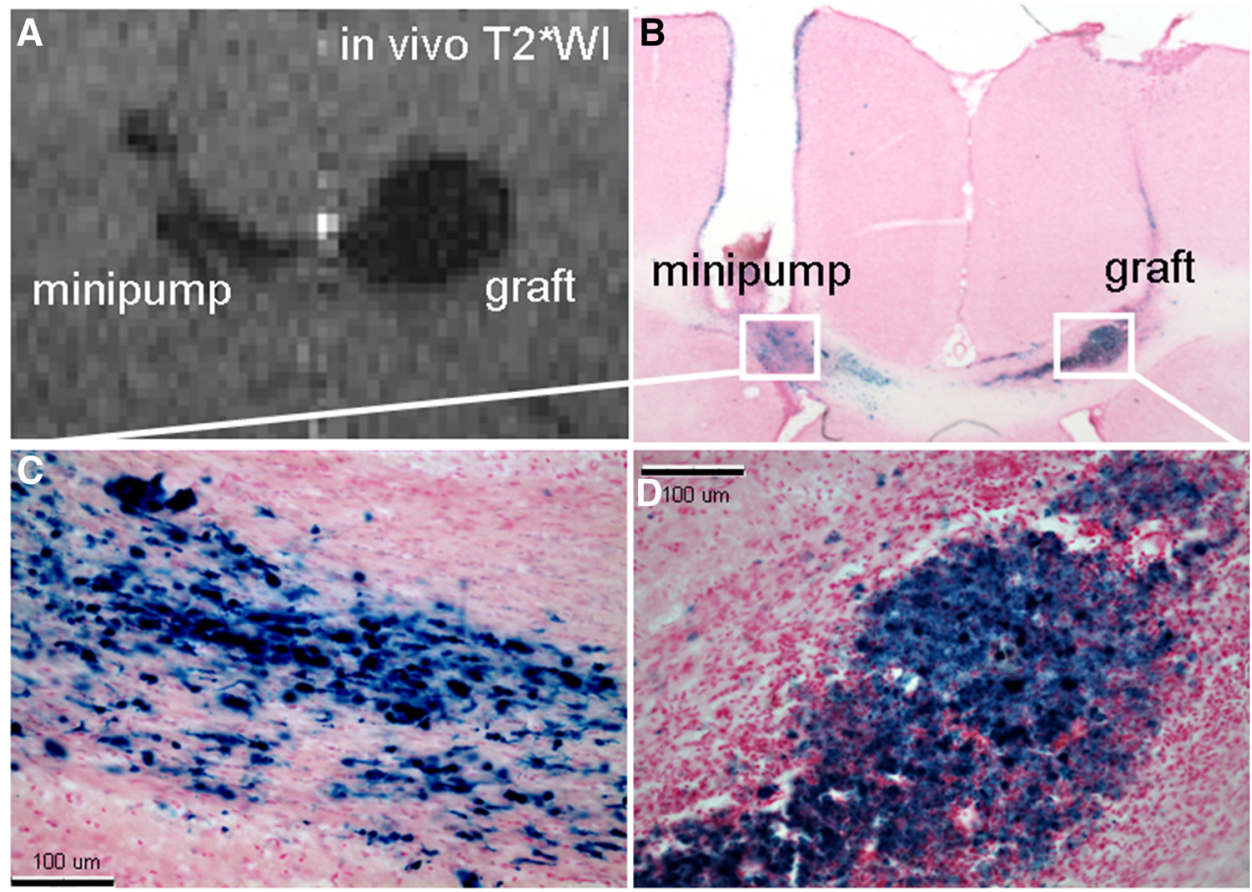

vehicle
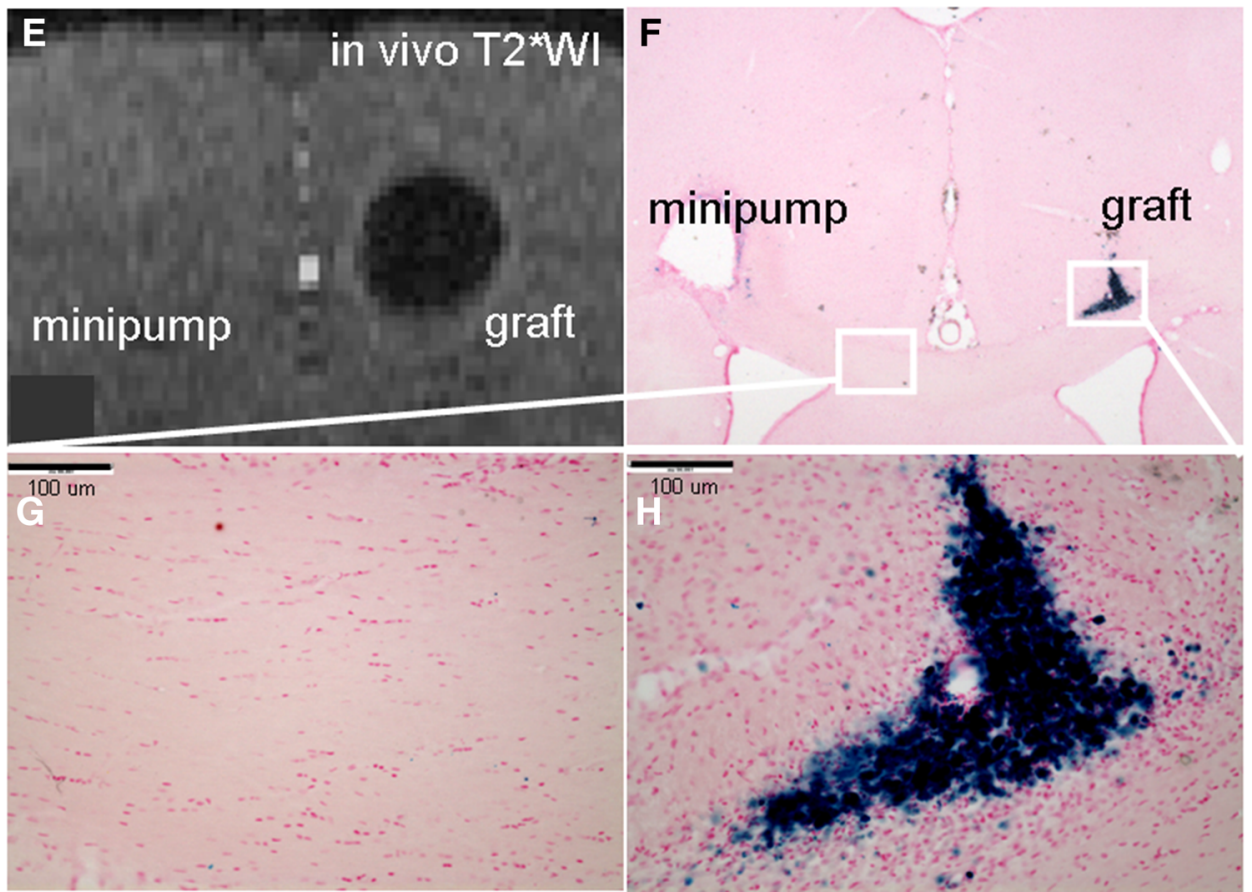

toward the target and of the graft are shown in Fig. 5c and $\mathrm{d}$, respectively. In the case of vehicle infusion, minimal migration was observed (Fig. 5e). PB-stained cells were mainly observed in the graft site as shown in Fig. 5f. Magnified views of the corpus callosum and the graft are shown in Fig. $5 \mathrm{~g}$ and $\mathrm{h}$, respectively.
Corroboration of the progenitor identity by nestin immunohistology

Figure $6 a$ shows the diagram of the infusion minipump, migratory path, and graft. The areas nearby the migratory path and graft marked by squares were photomicrographed. 

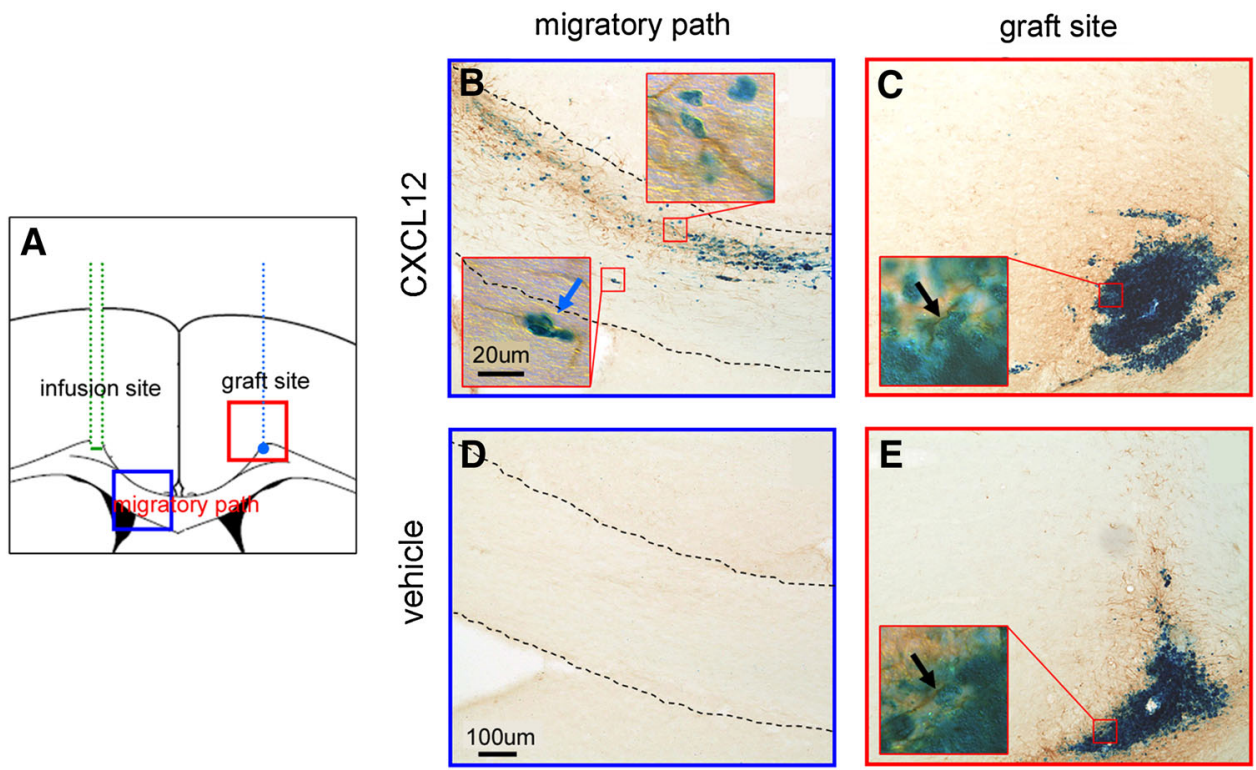

Fig. 6 Corroboration of the progenitor identity by nestin immunohistology. a The diagram of the infusion tube, migratory path, and graft. The areas nearby the graft and the migratory path marked by squares were photomicrographed. b A closer view of the migrating NPCs taken from the CXCL12-treated group. The NPCs were

Figure $6 \mathrm{~b}$ shows a closer view of the migrating NPCs. The NPCs were observed with both PB and nestin immunoreactivity. Figure $6 \mathrm{c}$ shows the graft treated with CXCL12 infusion. NPCs were positive for both nestin and PB. An additional photomicrograph at the off-center position of the graft is shown in Supplemental Fig S1, which reveals more distinct nestin immunoreactivity. In the vehicle-treated group, little migration occurred as shown in Fig. 6d. Figure 6e shows the graft treated with vehicle infusion. Positive staining was mainly seen at the graft site. Thus, neither PB staining nor nestin immunoreactivity was observed beyond the graft site. Regarding in vivo differentiation, it seems that the CXCL12 treatment regulated the fate of the transplanted NPCs, as shown in Supplemental Fig S2.

Non-adverse effects on NPCs caused by magnetic labeling

As shown in Fig. 7a, numerous PB-stained particles were found in the cytoplasm in the labeled NPCs. In Fig. 7b, MTT assays indicated no significant difference in cell proliferation between the unlabeled and labeled NPCs. Differentiation was also examined. Both the unlabeled and labeled NPCs exhibited similar capacities for cellular differentiation as shown in Fig. 7c and d, respectively. The iron content of the labeled NPCs was stained by DABenhanced PB as shown in Fig. 7e. Note that MPIO labeling tended to obstruct the fluorescence shown in Fig. 7d, observed with both PB and nestin immunoreactivity. $\mathbf{c}$ The NPCs of the graft treated with CXCL12 infusion were positive for both nestin and $\mathrm{PB}$. d Little migration occurred in the vehicle-treated group. e The graft view from the vehicle-treated group. Neither PB staining nor nestin immunoreactivity was observed beyond the graft site

leading to a misimpression of weaker immunoreactivity in the labeled NPCs. The results indicate that NPCs labeled with MPIO retained the ability to proliferate and differentiate.

\section{Discussion}

The present study investigated whether neurochemical attraction via CXCL12/CXCR4 signaling is responsible for the efficient migration along the CC. It was found that, in the spontaneous (vehicle) condition, little CXCL12 immunoreactivity was detected in the $\mathrm{CC}$, and this corresponded to the minimal migration seen during the monitoring period. The results indicate that NPCs had minimal mobility along the white matter tracts when the CXCL12 concentration was not up-regulated. By contrast, continuous CXCL12 infusion led to elevated CXCL12 levels in the $\mathrm{CC}$, and this significantly increased migration of the transplanted NPCs through the CC. Continuous CXCL12 administration at $60 \mathrm{ng} /$ day resulted in an averaged migration speed at $269 \pm 41 \mu \mathrm{m} /$ day or an averaged expansion rate of $115 \pm 35 \times 10^{6} \mu \mathrm{m}^{3} /$ day into the CC. CXCL12 augmented the average migration distance by a factor of up to 9 , and the overall volume by a factor of up to 29 compared to spontaneous migration. This indicates that CXCL12/CXCR4 signaling is a key mechanism whereby the white matter tracts are utilized by NPCs for efficient 
A

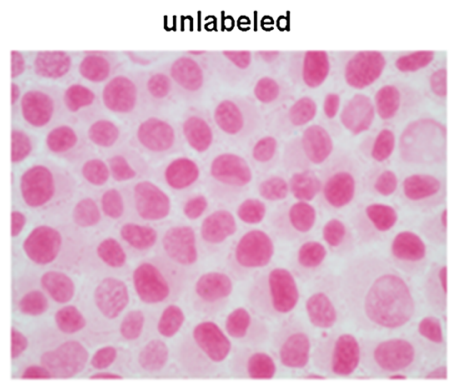

astrocyte

C

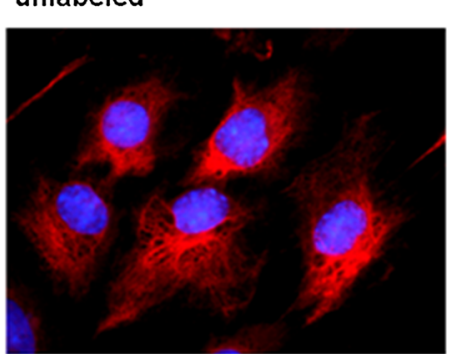

D

\section{labeled}

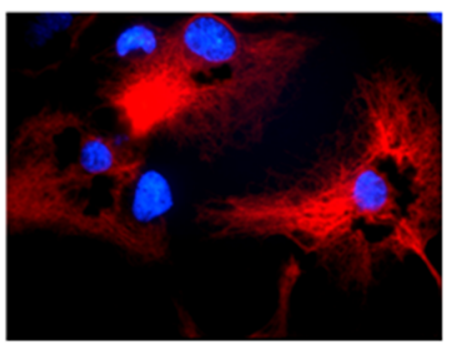

E

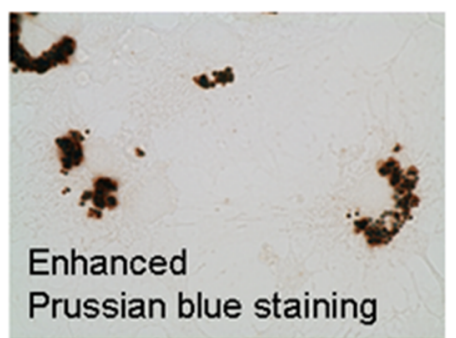

B MTT assay

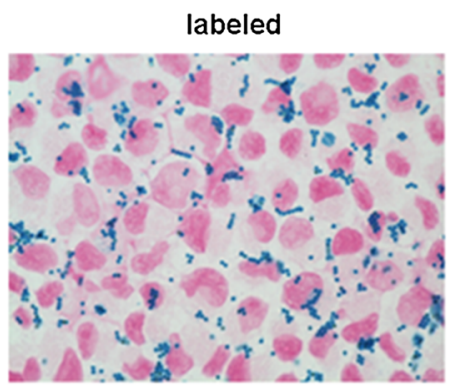

Differentiation assay

oligodendrocyte
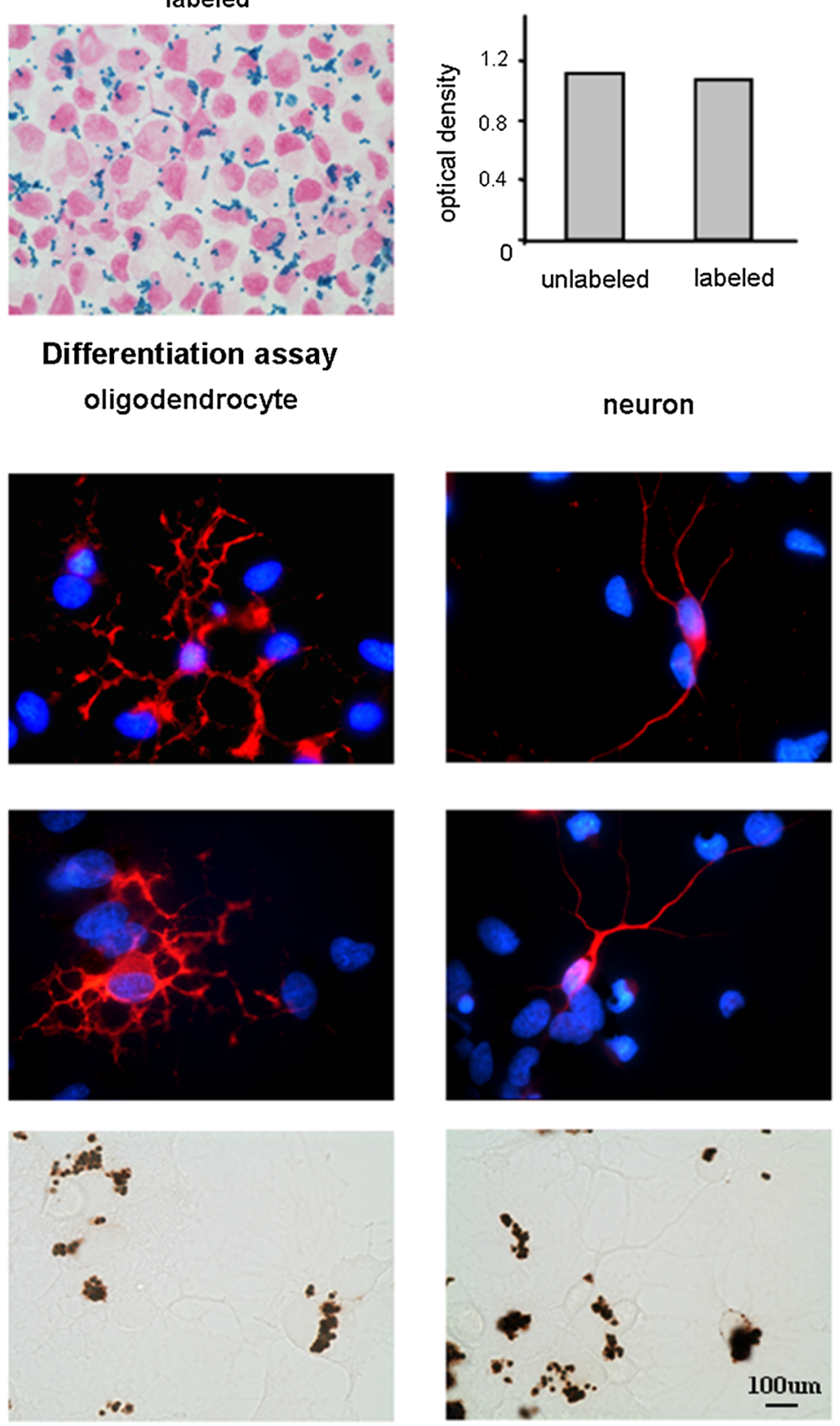

neuron
Fig. 7 NPCs labeled with MPIO retained the ability to proliferate and differentiate. a No positive PB staining was observed in the unlabeled NPCs while numerous PB-stained particles were found in the cytoplasm of the MPIO-labeled NPCs. b MTT assays indicated a similar proliferation capacity between the labeled and unlabeled NPCs. c Differentiation of the unlabeled NPCs into astrocytes,

movement. It is possible that high chemokine attraction in a structure with high directionality is an important combinational factor for efficient NPC migration across a long distance within the brain.

NPC migration into the white matter structures is well known in brain disorders involving axonal injuries or oligodendrocytes, and neurons. d The labeled NPCs preserved the differentiation capacity. $\mathbf{e}$ The iron content in the labeled NPCs was stained by diaminobenzidine-enhanced PB. The results indicated that NPCs labeled with MPIO retained the ability to proliferate and differentiate

demyelination (Nam et al. 2007; Zhang et al. 2003; Hoehn et al. 2002; Banisadr et al. 2011). In ischemic stroke, where white matter lesion is sometimes reported, engrafted NPCs migrated all the way across the callosal pathway to the ischemic lesioned area in a rat stroke model (Hoehn et al. 2002). The mean migration speed was estimated to range 
from $360 \mu \mathrm{m} /$ day (Kim et al. 2004) to 50-80 $\mu \mathrm{m} / \mathrm{h}$ (Zhang et al. 2003). In consistence with the findings, the expression of CXCL12 within the white matter structures was found to increase following hypoxic ischemic injury (Miller et al. 2005). In experimental autoimmune encephalomyelitis, NPCs engrafted in the lateral ventricle migrated up to $500 \mu \mathrm{m}$ through the white matter tracts (Ben-Hur et al. 2003). The migration speed of endogenous NPCs is approximately $50-80 \mu \mathrm{m} / \mathrm{h}$ in multiple sclerosis (Nam et al. 2007). Evidence has shown that the NPC migration through white matter in experimental autoimmune encephalomyelitis was also based upon the CXCL12/ CXCR4 signaling (Banisadr et al. 2011).

Nevertheless, NPCs migration into the white matter structures is not a scenario limited to white matter dysfunctions. That is, such migration is not necessarily intended for regional tissue repair. The present study and several previous reports indicate that migration into white matter also occurs in healthy subjects during the neonatal stage and in the adulthood. In these cases, the white matter structures may mainly serve as a migratory road for the cells. In neonatal rats, MRI revealed that endogenous NPCs labeled by MPIO migrated through white matter toward the cortex (Yang et al. 2009). In some cases, white matter in the vicinity of the subventricular zone is the only structure where endogenous migrating NPCs from the SVZ can be found (Suzuki and Goldman 2003). In the adult rat brain, there is a small degree of spontaneous migration along the white matter tracts. It was previously reported that NPCs engrafted in the corpus callosum migrated at the speed of 50-70 $\mu \mathrm{m} /$ day (Flexman et al. 2011). The present study reported the spontaneous migration speed along the callosal pathway being $29 \mu \mathrm{m} /$ day over the 7-day tracking period, lower than that reported earlier (Flexman et al. 2011). This difference may arise from variations in the experimental protocols, including the cell types, the labeling particles used, and the imaging resolution.

It needs to be addressed whether the dosage of CXCL12 at $60 \mathrm{ng} /$ day used in the present study is a physiologically relevant concentration. Previous in vitro studies indicated that CXCL12 concentrations at 100 to $1,000 \mathrm{ng} / \mathrm{mL}$ showed approximately 1.6 to 1.75 -folds of increases in migration in the Boyden chamber assay (Imitola et al. 2004). This concentration range was empirically tested in our pilot study. It was found that, to induce in vivo migration in the brain, equivalent dosages via continuous infusion are required. Lower concentrations produced little migratory effects as found in our pilot study. But the administered dose range was in a sharp contrast to the reported secreted levels of endogenous CXCL12 in brain injuries, which falls within the ranges of tens to hundreds of picogram per milliliter or milligram. The disparity may arise from the difference in the measurement. CXCL12 has a very short half-life $(25.8 \pm 4.6 \mathrm{~min}$ in the circulation $)$ (Rempel et al. 2000). Since CXCL12 secretion was measured at a fixed time point as the concentration to the tissue volume/mass, the level could be significantly underestimated when extrapolated to daily estimation. In addition, the short half-life may also affect the sensitivity and detectability of the measuring method, enzyme-linked immuno-sorbent assay (ELISA). If the factors of time and sensitivity loss are both considered, the dosage given in the present study could be within a physiologically reasonable range close to the secreted levels.

CXCL12 is also known for its influences on cell survival. To understand whether our approach produced effects on the survival of the engrafted NPCs, additional TUNEL staining was performed. But no significant difference in the percentage of TUNEL stained cells was observed between the two groups $(31.8 \pm 17.4 \%$ in CXCL12 vs. $28 \pm 10.2 \%$ in vehicle). The findings further indicate that the effects of CXCL12 on the engrafted NPCs were mainly on the migratory behavior.

One major limitation associated with the use of cellular MRI tracking is that gradual loss of iron oxide nanoparticles, which leads to lowered detectability (Berman et al. 2011). Cell division or spontaneous exocytosis is responsible for such labeling loss. In the present study, it was found that use of large-sized labeling particles alleviates this problem. Despite the criticisms regarding the use of iron particles in cell tracking, this approach was not found to be disappointing. The use of MPIO nanoparticles appeared to rectify some of the problems reported with the use of smaller nanoparticles such as superparamagnetic iron oxide (Shapiro et al. 2005; Neri et al. 2008). A very high spatial correspondence between nestin immunoreactivity and PB staining was observed. At the NPC graft site, the engrafted cells were heavily double stained with nestin and PB. The distribution of the staining matched well with the appearance on MRI. This indicates that most of the engrafted NPCs retained the MPIO labeling even after migrating away from the injection site. Some NPCs, observed without PB staining toward the tube site and thus not shown on MRI, could be either cells that lost labeling following engraftment, or endogenous NPCs originating from the subventricular zone or the subgranular zone. However, the occurrence of either case did not invalidate the utility of cellular MRI in tracking cells, since the correspondence between MRI and histological findings remained reasonably strong in most of the brain areas (Hoehn et al. 2002).

Related to the issue of loss of labeling, the second limitation of the cellular MRI tracking system is the MR signals supposedly arising from the labeled NPCs may be nonspecific. Upon the loss of labeling, the nanoparticles are either cleared from the region by scavenger cells or are 
taken up by surrounding cells. If this occurs, the hypointense signals appearing on the MRI may then represent behavior of different cells instead of those intended for observation (Berman et al. 2011). This could undermine the utility of MRI in cell tracking. However, the high correspondence among the hypointensities, PB staining, and most of the nestin staining indicates that this issue is of less concern in the present study. Very few PB-stained cells were nestin negative. Hence, at both the macro- and microscopic levels, cellular MRI appears to be a relatively reliable tool to monitor the migration of engrafted cells (Politi 2007; Lee et al. 2004; Mligiliche et al. 2005; Zhang et al. 2003, 2004).

\section{Conclusions}

The present study aimed to understand why the white matter tracts are an efficient route for NPC migration. Cellular MRI supports the importance of CXCL12/CXCR4 signaling in facilitating NPCs migrating along the CC. In addition to the mechanistic implications, the administration of CXCL12 into the white matter structures may be developed as a strategy to promote the migration of the transplanted NPC in the future.

Acknowledgments This work was supported by research grants from the National Science Council (NSC 98-2314-B-001-002-MY3).

Conflict of interest The authors declare no conflict of interest.

Open Access This article is distributed under the terms of the Creative Commons Attribution License which permits any use, distribution, and reproduction in any medium, provided the original author(s) and the source are credited.

\section{References}

Aboody KS, Brown A, Rainov NG, Bower KA, Liu S, Yang W, Small JE, Herrlinger U, Ourednik V, Black PM, Breakefield XO, Snyder EY (2000) Neural stem cells display extensive tropism for pathology in adult brain: evidence from intracranial gliomas. Proc Natl Acad Sci USA 97(23):12846-12851. doi:10.1073/ pnas.97.23.12846

Assaf Y, Pasternak O (2008) Diffusion tensor imaging (DTI)-based white matter mapping in brain research: a review. J Mol Neurosci 34(1):51-61. doi:10.1007/s12031-007-0029-0

Banisadr G, Frederick TJ, Freitag C, Ren D, Jung H, Miller SD, Miller RJ (2011) The role of CXCR4 signaling in the migration of transplanted oligodendrocyte progenitors into the cerebral white matter. Neurobiol Dis 44(1):19-27. doi:10.1016/j.nbd. 2011.05.019

Belmadani A, Tran PB, Ren D, Assimacopoulos S, Grove EA, Miller RJ (2005) The chemokine stromal cell-derived factor-1 regulates the migration of sensory neuron progenitors. J Neurosci 25(16):3995-4003
Belmadani A, Tran PB, Ren D, Miller RJ (2006) Chemokines regulate the migration of neural progenitors to sites of neuroinflammation. J Neurosci 26(12):3182-3191

Ben-Hur T, Einstein O, Mizrachi-Kol R, Ben-Menachem O, Reinhartz E, Karussis D, Abramsky O (2003) Transplanted multipotential neural precursor cells migrate into the inflamed white matter in response to experimental autoimmune encephalomyelitis. Glia 41(1):73-80

Berman SC, Galpoththawela C, Gilad AA, Bulte JW, Walczak P (2011) Long-term MR cell tracking of neural stem cells grafted in immunocompetent versus immunodeficient mice reveals distinct differences in contrast between live and dead cells. Magn Reson Med 65(2):564-574. doi:10.1002/mrm.22613

Bhattacharyya BJ, Banisadr G, Jung H, Ren D, Cronshaw DG, Zou Y, Miller RJ (2008) The chemokine stromal cell-derived factor-1 regulates GABAergic inputs to neural progenitors in the postnatal dentate gyrus. J Neurosci 28(26):6720-6730

Flexman JA, Cross DJ, Tran LN, Sasaki T, Kim Y, Minoshima S (2011) Quantitative analysis of neural stem cell migration and tracer clearance in the rat brain by MRI. Mol Imaging Biol 13(1):104-111

Hoehn M, Kustermann E, Blunk J, Wiedermann D, Trapp T, Wecker S, Focking M, Arnold H, Hescheler J, Fleischmann BK, Schwindt W, Buhrle C (2002) Monitoring of implanted stem cell migration in vivo: a highly resolved in vivo magnetic resonance imaging investigation of experimental stroke in rat. Proc Natl Acad Sci USA 99(25):16267-16272

Imitola J, Raddassi K, Park KI, Mueller FJ, Nieto M, Teng YD, Frenkel D, Li J, Sidman RL, Walsh CA, Snyder EY, Khoury SJ (2004) Directed migration of neural stem cells to sites of CNS injury by the stromal cell-derived factor $1 \mathrm{alph} / \mathrm{CXC}$ chemokine receptor 4 pathway. Proc Natl Acad Sci USA 101(52):1811718122. doi:10.1073/pnas.0408258102

Itoh T, Satou T, Ishida H, Nishida S, Tsubaki M, Hashimoto S, Ito H (2009) The relationship between SDF-1alpha/CXCR4 and neural stem cells appearing in damaged area after traumatic brain injury in rats. Neurol Res 31(1):90-102

Kim DE, Schellingerhout D, Ishii K, Shah K, Weissleder R (2004) Imaging of stem cell recruitment to ischemic infarcts in a murine model. Stroke 35(4):952-957. doi:10.1161/01.STR.0000120308. 21946.5D

Lee IH, Bulte JW, Schweinhardt P, Douglas T, Trifunovski A, Hofstetter C, Olson L, Spenger C (2004) In vivo magnetic resonance tracking of olfactory ensheathing glia grafted into the rat spinal cord. Exp Neurol 187(2):509-516

Liapi A, Pritchett J, Jones O, Fujii N, Parnavelas JG, Nadarajah B (2008) Stromal-derived factor 1 signalling regulates radial and tangential migration in the developing cerebral cortex. Dev Neurosci 30(1-3): 117-131

Miller JT, Bartley JH, Wimborne HJ, Walker AL, Hess DC, Hill WD, Carroll JE (2005) The neuroblast and angioblast chemotaxic factor SDF-1 (CXCL12) expression is briefly up regulated by reactive astrocytes in brain following neonatal hypoxicischemic injury. BMC Neurosci 6:63. doi:10.1186/1471-22026-63

Mligiliche NL, Xu Y, Matsumoto N, Idel C (2005) Survival of neural progenitor cells from the subventricular zone of the adult rat after transplantation into the host spinal cord of the same strain of adult rat. Anat Sci Int 80(4):229-234

Nam SC, Kim Y, Dryanovski D, Walker A, Goings G, Woolfrey K, Kang SS, Chu C, Chenn A, Erdelyi F, Szabo G, Hockberger P, Szele FG (2007) Dynamic features of postnatal subventricular zone cell motility: a two-photon time-lapse study. J Comp Neurol 505(2):190-208. doi:10.1002/cne.21473

Neri M, Maderna C, Cavazzin C, Deidda-Vigoriti V, Politi LS, Scotti G, Marzola P, Sbarbati A, Vescovi AL, Gritti A (2008) Efficient 
in vitro labeling of human neural precursor cells with superparamagnetic iron oxide particles: relevance for in vivo cell tracking. Stem Cells (Dayton, Ohio) 26(2):505-516

Peng H, Kolb R, Kennedy JE, Zheng J (2007) Differential expression of CXCL12 and CXCR4 during human fetal neural progenitor cell differentiation. J Neuroimmune Pharmacol 2(3):251-258

Politi LS (2007) MR-based imaging of neural stem cells. Neuroradiology 49(6):523-534

Rempel SA, Dudas S, Ge S, Gutierrez JA (2000) Identification and localization of the cytokine SDF1 and its receptor, CXC chemokine receptor 4 , to regions of necrosis and angiogenesis in human glioblastoma. Clin Cancer Res 6(1):102-111

Robin AM, Zhang ZG, Wang L, Zhang RL, Katakowski M, Zhang L, Wang Y, Zhang C, Chopp M (2006) Stromal cell-derived factor 1alpha mediates neural progenitor cell motility after focal cerebral ischemia. J Cereb Blood Flow Metab 26(1):125-134

Shapiro EM, Skrtic S, Koretsky AP (2005) Sizing it up: cellular MRI using micron-sized iron oxide particles. Magn Reson Med 53(2):329-338

Shin JW, Lee JK, Lee JE, Min WK, Schuchman EH, Jin HK, Bae JS (2011) Combined effects of hematopoietic progenitor cell mobilization from bone marrow by granulocyte colony stimulating factor and AMD3100 and chemotaxis into the brain using stromal cell-derived factor-1alpha in an Alzheimer's disease mouse model. Stem Cells (Dayton, Ohio) 29(7):1075-1089. doi:10.1002/stem.659

Shyu WC, Lin SZ, Yen PS, Su CY, Chen DC, Wang HJ, Li H (2008) Stromal cell-derived factor-1 alpha promotes neuroprotection, angiogenesis, and mobilization/homing of bone marrow-derived cells in stroke rats. J Pharmacol Exp Ther 324(2):834-849

Stumm R, Hollt V (2007) CXC chemokine receptor 4 regulates neuronal migration and axonal pathfinding in the developing nervous system: implications for neuronal regeneration in the adult brain. J Mol Endocrinol 38(3):377-382
Suzuki SO, Goldman JE (2003) Multiple cell populations in the early postnatal subventricular zone take distinct migratory pathways: a dynamic study of glial and neuronal progenitor migration. J Neurosci 23(10):4240-4250

Takeuchi H, Natsume A, Wakabayashi T, Aoshima C, Shimato S, Ito M, Ishii J, Maeda Y, Hara M, Kim SU, Yoshida J (2007) Intravenously transplanted human neural stem cells migrate to the injured spinal cord in adult mice in an SDF-1- and HGFdependent manner. Neurosci Lett 426(2):69-74. doi:10.1016/j. neulet.2007.08.048

Tiveron MC, Rossel M, Moepps B, Zhang YL, Seidenfaden R, Favor J, Konig N, Cremer H (2006) Molecular interaction between projection neuron precursors and invading interneurons via stromal-derived factor 1 (CXCL12)/CXCR4 signaling in the cortical subventricular zone/intermediate zone. J Neurosci 26(51):13273-13278

Yang J, Liu J, Niu G, Chan KC, Wang R, Liu Y, Wu EX (2009) In vivo MRI of endogenous stem/progenitor cell migration from subventricular zone in normal and injured developing brains. NeuroImage 48(2):319-328. doi:10.1016/j.neuroimage.2009.06. 075

Zhang SC, Wernig M, Duncan ID, Brustle O, Thomson JA (2001) In vitro differentiation of transplantable neural precursors from human embryonic stem cells. Nat Biotechnol 19(12):1129-1133. doi:10.1038/nbt1201-1129

Zhang ZG, Jiang Q, Zhang R, Zhang L, Wang L, Zhang L, Arniego P, Ho KL, Chopp M (2003) Magnetic resonance imaging and neurosphere therapy of stroke in rat. Ann Neurol 53(2):259-263

Zhang Z, Jiang Q, Jiang F, Ding G, Zhang R, Wang L, Zhang L, Robin AM, Katakowski M, Chopp M (2004) In vivo magnetic resonance imaging tracks adult neural progenitor cell targeting of brain tumor. NeuroImage 23(1):281-287 